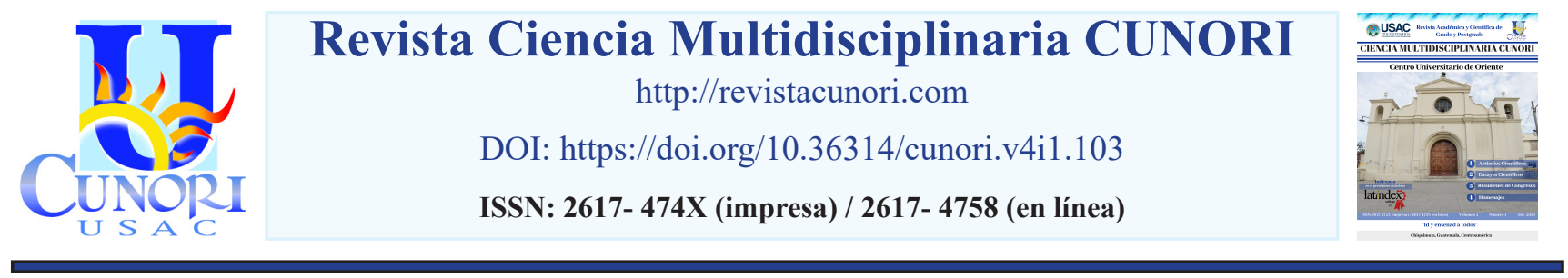

Como citar el artículo

Fajardo, I., García, L y Retana, R. (2020). Cribado de trastorno por déficit de atención e hiperactividad en estudiantes de medicina. Revista Ciencia Multidisciplinaria CUNORI, 4(1), 1-6. DOI: https://doi.org/10.36314/cunori.v4i1.103

\title{
Cribado de trastorno por déficit de atención e hiperactividad en estudiantes de medicina
}

\section{Screening for attention deficit hyperactivity disorder in medical students}

\author{
Iván Fajardo*, Luis García y Ronaldo Retana \\ Centro Universitario de Oriente (CUNORI), Universidad de San Carlos de Guatemala
}

Recibido: 30 de mayo de 2019 / Revisión: 29 de noviembre de 2019 / Aceptado: 3 de febrero de 2020

Disponible en internet el 29 de mayo de 2020

\author{
*Autor para correspondencia. \\ Correo electrónico: ivanfajardo1994@gmail.com \\ Resumen
}

- 1 trastorno por déficit de atención e hiperactividad (TDAH) se consideraba un desorden del neurodesarrollo exclusivamente Cde la infancia, pero en la actualidad se conoce que puede persistir en la edad adulta, pero dificulta identificarlo porque la manifestación de los síntomas difiere en adultos. Esta patología afecta el ámbito laboral, académico, profesional, familiar y social del individuo afectado, lo que les impide desarrollar al máximo su potencial. Se realizó un estudio de carácter descriptivo transversal, el cual determinó la probabilidad de presentar TDAH y características sociodemográficas en quienes tenían probabilidad de TDAH, en estudiantes de la carrera de Médico y Cirujano del Centro Universitario de Oriente -CUNORI- de Chiquimula, Guatemala, durante febrero del año 2019, empleando como instrumento de cribado el cuestionario autoinformado de cribado de TDAH del adulto versión 1.1 (ASRS v1.1). Fue tomado para el estudio todo el universo de población para que el estudio fuera significativo, estos fueron 205 estudiantes que cursaban cuarto, quinto y sexto año académico. De los 205 estudiantes el 10.7\% mostró probabilidad de presentar TDAH. Las características sociodemográficas en quienes tenían probabilidad del trastorno fueron: cursar cuarto año $59.1 \%$, edad de 21 a 22 años $40.9 \%$, consumo de tabaco $27.3 \%$, sexo masculino $54.5 \%$, otra carrera universitaria cursada anteriormente $4.5 \%$, repitencia de año o rotación $72.7 \%$ de los casos. En conclusión, la probabilidad de presentar TDAH en los estudiantes de medicina fue de $10.7 \%$, por lo que debe realizarse otras investigaciones para detección de casos de TDAH y darles seguimiento.

Palabras clave: trastorno por déficit de atención e hiperactividad (TDAH), cribado

\begin{abstract}
A ttention deficit hyperactivity disorder (ADHD) was considered a neurodevelopmental disorder exclusively in childhood, but it is now known that it can persist in adulthood, but it is difficult to identify it because the manifestation of symptoms differs in adults. This pathology affects the work, academic, professional, family and social environment of the affected individual, which prevents them from developing their full potential. A cross-sectional descriptive study was conducted, which determined the probability of presenting ADHD and sociodemographic characteristics in those who had ADHD probability, in students of the Medical and Surgery career of the University Center of Oriente -CUNORI- of Chiquimula, Guatemala, during February of the year 2019, using as a screening instrument the self-report questionnaire for adult ADHD screening version 1.1 (ASRS v1.1). The entire universe of population was taken for the study so that the study was significant, these were 205 students who were studying fourth, fifth and sixth academic year. Of the 205 students, $10.7 \%$ showed a probability of presenting ADHD. The sociodemographic characteristics in those who had probability of the disorder were: to study fourth year 59.1\%, age from 21 to 22 years $40.9 \%$, tobacco consumption $27.3 \%$, male gender $54.5 \%$, another university career previously completed $4.5 \%$, year repetition or rotation
\end{abstract}


$72.7 \%$ of cases. In conclusion, the probability of presenting ADHD in medical students was $10.7 \%$, so other investigations should be conducted to detect ADHD cases and follow up.

Keywords: attention deficit hyperactivity disorder (ADHD), screening

\section{Introducción}

El trastorno por déficit de atención e hiperactividad (TDAH) es caracterizado por la persistencia de patrón de inatención y/o hiperactividad-impulsividad, cuya condición afecta el funcionamiento y desarrollo en diversos aspectos de la vida del individuo (APA, 2014). Se estima que en más de la mitad de los niños con TDAH el desorden puede continuar en la adolescencia y la edad adulta (Caye et al. 2016). Los adultos pueden estar viviendo con TDAH sin darse cuenta ellos mismos y las personas que le rodean, ya que los síntomas se pueden confundir con el estrés (Adler et al. 2005). Los síntomas se asocian frecuentemente a otros problemas como adicción a sustancias, trastornos de sueño, humor, ansiedad y personalidad entre otros (Kooij et al. 2010). En el ámbito profesional y laboral, se ha documentado afección en la funcionalidad del individuo presentando problemas de coordinación motora, memoria laboral, planeación y anticipación, también experimentan dificultades en fluidez verbal, orientación del esfuerzo, empleo de estrategias de organización y autoregulación de emociones de gran impacto (Adamou et al. 2013). La Organización Mundial de la Salud (OMS) diseñó el cuestionario autoinformado de cribado de TDAH del adulto versión 1.1 (ASRS v1.1), el cual consta de 6 preguntas dirigidas para adultos con rasgos de TDAH e identifica la probabilidad de que un adulto presente TDAH, dicha escala no es diagnóstica y se necesita la evaluación de un médico capacitado para realizar el diagnóstico (Harvard Medical School s.f.). El ASRS v1.1 fue validado por la OMS para su uso en español, ya que presenta excelentes características psicométricas haciendo su utilización eficaz para detectar casos probables con TDAH (Ramos-Quiroga, 2009).

\section{Materiales y métodos}

Para el estudio se tomo a 205 estudiantes de cuarto, quinto y sexto año académico, de la carrera de Médico y Cirujano del Centro Universitario de Oriente -CUNORI- durante febrero del año 2019. Para la recolección de datos fue utilizada una boleta con información aportada por el estudiante, dividida en dos partes, la primera parte recopiló características sociodemográficas tales como rotación que cursan, edad, sexo, consumo de tabaco, repitencia de año o rotación, carrera universitaria cursada anteriormente. La segunda parte constó del cuestionario autoinformado de cribado de TDAH del adulto versión 1.1 (ASRS v1.1), compuesta por 6 items que evalúan síntomas de TDAH. Los datos obtenidos se ingresaron y analizaron en Excel $2016^{\mathrm{TM}}$ utilizando estadística descriptiva, mostrando los resultados mediante tablas de información.

\section{Resultados}

Tabla No. 1. Distribución de estudiantes evaluados a través del cuestionario autoinformado de cribado del trastorno por déficit de atención e hiperactividad del adulto- v1.1, con probabilidad de TDAH, febrero del año 2019. 


\begin{tabular}{ccc}
\hline Probabilidad de TDAH & Frecuencia & Porcentaje (\%) \\
No & 183 & 89.3 \\
$\mathrm{Si}$ & 22 & 10.7 \\
Total & 205 & 100 \\
\hline
\end{tabular}

La probabilidad de TDAH observada en estudiantes evaluados a través del cuestionario autoinformado de cribado del trastorno por déficit de atención e hiperactividad del adulto-v1.1 presenta una frecuencia de $10.7 \%$ (22) para quienes si tienen probabilidad de TDAH y de $89.3 \%$ (183) para quienes no tienen probabilidad de TDAH.

Tabla No. 2. Distribución de estudiantes evaluados a través del cuestionario autoinformado de cribado del trastorno por déficit de atención e hiperactividad del adulto- v1.1, con probabilidad de TDAH, según año académico en curso, febrero del año 2019.

\begin{tabular}{ccc}
\hline Año académico & Frecuencia & Porcentaje (\%) \\
Cuarto & 13 & 59.1 \\
Quinto & 3 & 13.6 \\
Sexto & 6 & 27.3 \\
Total & 22 & 100 \\
\hline
\end{tabular}

En los estudiantes que presentan probabilidad de TDAH, se observa que el año académico que presenta mayor frecuencia de poder manifestar esta enfermedad es cuarto año compuesto por $59.1 \%$ (13) y sucesivo de este sexto año con $27.3 \%$ (6).

Tabla No. 3. Distribución de estudiantes evaluados a través del cuestionario autoinformado de cribado del trastorno por déficit de atención e hiperactividad del adulto- v1.1, con probabilidad de tener TDAH, según edad, febrero del año 2019.

\begin{tabular}{ccc}
\hline Edad (años) & Frecuencia & Porcentaje (\%) \\
$19-20$ & 1 & 4.5 \\
$21-22$ & 9 & 40.9 \\
$23-24$ & 5 & 22.7 \\
$25-26$ & 5 & 22.7 \\
$27-28$ & 1 & 4.5 \\
$29-30$ & 0 & 0 \\
$31-32$ & 1 & 4.5 \\
$\geq 33$ & 0 & 0 \\
Total & 22 & 99.8 \\
\hline
\end{tabular}

En los estudiantes con probabilidad de TDAH, se observa que la edad en rango con mayor frecuencia de presentar TDAH es de 21 a 22 años representado por $40.9 \%$ (9), seguido de este 23 a 24 años y de 25 a 26 años con $22.7 \%$ para cada uno (5 cada uno). 
Tabla No. 4. Distribución de estudiantes evaluados a través del cuestionario autoinformado de cribado del trastorno por déficit de atención e hiperactividad del adulto- v1.1, con probabilidad de TDAH, según sexo, febrero del año 2019.

\begin{tabular}{ccc}
\hline Sexo & Frecuencia & Porcentaje (\%) \\
Femenino & 10 & 45.5 \\
Masculino & 12 & 54.5 \\
Total & 22 & 100 \\
\hline
\end{tabular}

Se observa que el sexo que presenta mayor frecuencia en estudiantes con probabilidad de TDAH es masculino representado por $54.5 \%$ (12).

Tabla No. 5. Distribución de estudiantes evaluados a través del cuestionario autoinformado de cribado del trastorno por déficit de atención e hiperactividad del adulto- v1.1, con probabilidad de tener TDAH, según consumo de tabaco, febrero del año 2019.

\begin{tabular}{ccc}
\hline Consumo de tabaco & Frecuencia & Porcentaje (\%) \\
No & 16 & 72.7 \\
$\mathrm{Si}$ & 6 & 27.3 \\
Total & 22 & 100 \\
\hline
\end{tabular}

El consumo de tabaco observado en estudiantes con probabilidad de TDAH, muestra una frecuencia para quienes no consumen tabaco de $72.7 \%$ (16) y de $27.3 \%$ (6) para quienes consumen.

Tabla No. 6. Distribución de estudiantes evaluados a través del cuestionario autoinformado de cribado del trastorno por déficit de atención e hiperactividad del adulto- v1.1, con probabilidad de tener TDAH, según repitencia de año o rotación, febrero del año 2019.

\begin{tabular}{ccc}
\hline Repitencia de año o rotación & Frecuencia & Porcentaje (\%) \\
No & 6 & 27.3 \\
Si & 16 & 72.7 \\
Total & 22 & 100 \\
\hline
\end{tabular}

La repitencia de año o rotación observada en estudiantes con probabilidad de TDAH, señala una frecuencia para quienes si repitieron de $72.7 \%$ (16) y de $27.3 \%$ (6) para quienes no han repetido. 
Tabla No. 7. Distribución de estudiantes evaluados a través del cuestionario autoinformado de cribado del trastorno por déficit de atención e hiperactividad del adulto- v1.1, con probabilidad de tener TDAH, según carrera universitaria cursada anteriormente, febrero del año 2019.

\begin{tabular}{ccc}
\hline $\begin{array}{c}\text { Carrera universitaria cursada } \\
\text { anteriormente }\end{array}$ & Frecuencia & Porcentaje (\%) \\
$\mathrm{No}$ & 21 & 95.5 \\
$\mathrm{Si}$ & 1 & 4.5 \\
Total & 22 & 100 \\
\hline
\end{tabular}

La frecuencia de carrera universitaria cursada previamente observada en estudiantes con probabilidad de TDAH, señala $4.5 \%$ (1).

\section{Discusión}

De un total de 205 estudiantes evaluados, se estableció que el 10.7\% tiene probabilidad de presentar TDAH, ya que el ASRS v1.1 determina probabilidad, para confirmar el diagnóstico los estudiantes deben ser sometidos a una evaluación psiquiátrica. Se determinaron las características sociodemográficas en quienes tienen probabilidad de TDAH, las cuales son: cursando cuarto año $59.1 \%$, edad de 21 a 22 años $40.9 \%$, consumo de tabaco $27.3 \%$, sexo masculino $54.5 \%$, otra carrera universitaria cursada anteriormente $4.5 \%$, repitencia de año o rotación $72.7 \%$.

\section{Agradecimientos}

Al Dr. Ronaldo Armando Retana Albanés, coordinador de la carrera de Médico y Cirujano del Centro Universitario de Oriente por permitir la realización de este tema de investigación en la población estudiantil y al Comité Organizador de Trabajos de Graduación de Medicina por aprobar el tema a investigar. Al Dr. Luis Alfredo García Lima, especialista en medicina interna, por su asesoría y dedicación brindada durante la realización de la investigación. Al Dr. Edvin Danilo Mazariegos Albanés, especialista en medicina interna, por su apoyo en la revisión del presente manuscrito.

\section{Referencias}

Adamou, M. 2013. Occupational issues of adults with ADHD, 13(59), 2-4. https://doi.org/10.1186/1471 $-244 \mathrm{X}-13-59$

Adler, L. 2005. Escala de autodetección del trastorno por déficit de atención - hiperactividad (TDAH) del adulto-V1.1 (ASRSV1.1) - detección: de la encuesta diagnóstica internacional compuesta, 1-4. Recuperado de https://www.hcp.med.harvard.edu/ncs/ftpdir/adhd/6Q_Spanish\%20(for\%20US\%20 and\%20Mexico)_final.pdf 
APA (American Psiqhiatric Association). 2014. Manual diagnóstico y estadístico de los trastornos mentales DSM-5, 59- 65. Recuperado de: https://www.academia.edu/17057730/Manual_Diagnostico_y_Estadistico_de_los_Trastornos_Mentales_DSM-5_APA_5ed_booksmedicos

Caye, A. 2016. Predictors of persistence of ADHD into adulthood: a systematic review of the literature and meta-analysis, 25(11), 1151-1159. Recuperado de: https://www.researchgate.net/publication/299479933_Predictors_of_persistence_of_ADHD_into_adulthood_a_systematic_review_of_ the_literature_and_meta-analysis

Harvard Medical School. s.f. Adult ADHD Self-Report Scale (ASRS) version 1.1 background information, 2. Recuperado de: https://www.hcp.med.harvard.edu/ncs/ftpdir/adhd/background_memo_rev. pdf

Kooij, SJJ. 2010. European consensus statement on diagnosis and treatment of adult ADHD: the european network adult ADHD, 10(67), 1-24. Recuperado de https://www.ncbi.nlm.nih.gov/pmc/articles/ PMC2942810/

Ramos-Quiroga, JA. 2009. TDAH en adultos: factores genéticos, evaluación y tratamiento farmacológico, 258, Recuperado de: https://www.tdx.cat/bitstream/ handle/10803/5585/jarq1de1.pdf

\section{Sobre el autor}

\section{Iván Antonio Fajardo Villeda}

Es Médico y Cirujano egresado del Centro Universitario de Oriente CUNORI de Universidad de San Carlos de Guatemala.

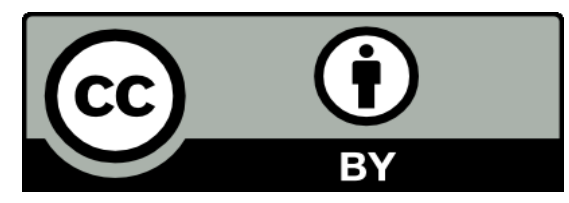

Este texto está protegido por una licencia CreativeCommons 4.0.

Usted es libre para compartir, copiar y redistribuir el material en cualquier medio o formato y adaptar el documento, remezclar, transformar y crear a partir del material para cualquier propósito, incluso comercialmente, siempre que cumpla la condición de atribución: usted debe reconocer el crédito de una obra de manera adecuada, proporcionar un enlace a la licencia, e indicar si se han realizado cambios. Puede hacerlo en cualquier forma razonable, pero no de forma tal que sugiera que tiene el apoyo del licenciante o lo recibe por el uso que hace. 